\title{
LOWER CENTRAL DEPTH IN FINITELY GENERATED SOLUBLE-BY-FINITE GROUPS
}

\author{
by JOHN C. LENNOX
}

(Received 28 March, 1977)

We say that a group $G$ has finite lower central depth (or simply, finite depth) if the lower central series of $G$ stabilises after a finite number of steps.

In [1], we proved that if $G$ is a finitely generated soluble group in which each two generator subgroup has finite depth then $G$ is a finite-by-nilpotent group. Here, in answer to a question of $\mathbf{R}$. Baer, we prove the following stronger version of this result.

THEOREM. Suppose that $G$ is a finitely generated soluble-by-finite group in which every subgroup of the form $\left\langle x, x^{y}\right\rangle, x$ and $y$ in $G$, has finite lower central depth. Then $G$ is finiteby-nilpotent.

It is easy to deduce, by methods analogous to those in [1], that the result also holds for the classes of finitely generated linear groups and finitely generated hyper-(abelian-byfinite) groups.

Proof. Arguing as in the first part of the proof of Theorem 3 of [1], we may assume that $G$ has a residually nilpotent soluble subgroup $N$ of finite index.

Let $x, y$ be elements of $N$. Then $X=\left\langle x, x^{y}\right\rangle$ is residually nilpotent and has finite lower central depth by hypothesis. Hence $X$ is nilpotent. Let $\bar{N}$ be any finite homomorphic image of $N$. Then the image $\bar{X}$ of $X$ in $\bar{N}$ is nilpotent and hence, if $\alpha$ and $\beta$ are the images of $x$ and $y$ in $\bar{N}$, we have that there exists a positive integer $n$ such that the repeated commutator

$$
\left[\alpha^{\beta},{ }_{n} \alpha\right]=1 \text {. }
$$

Since $\bar{N}$ is finite and $y$ is arbitrary it follows from a result of Peng [2] (see also [3, 7.22]) that the normal closure of $\alpha$ in $\bar{N}$ is nilpotent. Since $x$ is arbitrary and $\bar{N}$ is finite, it follows that $\bar{N}$ is nilpotent.

Thus $N$ has all of its finite homomorphic images nilpotent and is finitely generated since it is of finite index in a finitely generated group. It now follows from a theorem of Robinson $[3,10.51]$ that $N$ is nilpotent.

The fact that $G$ is finite-by-nilpotent follows as in the last three paragraphs of the proof of Theorem 3 of $[\mathbf{1}]$.

\section{REFERENCES}

1. J. C. Lennox, Finitely generated soluble groups in which all subgroups have finite lower central depth, Bull. London Math. Soc. 7 (1975), 273-278.

Glasgow Math. J. 19 (1978) 153-154. 
2. T. A. Peng, Engel elements of groups with maximal condition on abelian subgroups, Nanta Math. 1 (1966), 23-28.

3. D. J. S. Robinson, Finiteness conditions and generalised soluble groups, Vol II (Springer, 1972).

Department of Pure Mathematics

University COLlege

P.O. Box 78

CardifF CF1 $1 \mathrm{XL}$ 